\title{
ACOMPANHAMENTO LONGITUDINAL DO MANEJO DE SINTOMAS EM SERVIÇO ESPECIALIZADO DE CUIDADOS PALIATIVOS ONCOLÓGICOS
}

Karine Marques Costa dos Reis ${ }^{1}$ Cristine Alves Costa de Jesus ${ }^{1}$ https://orcid.org/0000-0002-2025-7247
https://orcid.org/0000-0002-8638-4468

Objetivo: Analisar a funcionalidade e os sintomas de pacientes oncológicos em fim de vida durante internação em unidade especializada em cuidados paliativos. Método: Trata-se de avaliação longitudinal da admissão até o óbito, com monitorização semanal da funcionalidade e score de sintomas, de 59 participantes. Utilizou-se o teste paramétrico Wilcoxon pareado, para avaliação da mediana dos scores de sintomas e funcionalidade com nível de significância de $5 \%$. Resultados: A amostra do estudo consistiu em indivíduos do sexo feminino, com idade média de 60 anos e funcionalidade limitada. Predominou o diagnóstico de câncer do trato digestório. Quanto a avaliação dos sintomas e funcionalidade, considerando a segunda e terceira semana de internação observou-se melhora do score dor, cansaço e tristeza, como também da função. Conclusão: Apesar de internação tardia e com grande extensão da doença, o participante apresentou melhora de alguns sintomas sugerindo o benefício dos cuidados paliativos. Em unidade especializada, houve melhora dos sintomas dor, cansaço e tristeza, com também da funcionalidade dos participantes em duas semanas de internação. Assim, esses achados corroboram quanto a importância do cuidado baseado no conforto e auxiliará na elaboração de políticas públicas e no planejamento do cuidado do enfermeiro.

Descritores: Cuidados Paliativos; Sinais e Sintomas; Neoplasias; Enfermagem.

\section{LONGITUDINAL FOLLOW-UP OF THE MANAGEMENT OF SYMPTOMS IN SPECIALIZED PALLIATIVE ONCOLOGICAL CARE SERVICE}

Objective: To analyze the functionality and symptoms of end-of-life cancer patients during hospitalization in a specialized unit in palliative care. Method: This is a longitudinal assessment from admission to death, with weekly monitoring of functionality and symptom score, of 59 participants. The paired Wilcoxon parametric test was used to assess the median of symptom scores and functionality with a significance level of $5 \%$. Results: The study sample consisted of female individuals, with an average age of 60 years and limited functionality. The diagnosis of cancer of the digestive tract predominated. Regarding the assessment of symptoms and functionality, considering the second and third week of hospitalization, an improvement was noted in the pain, tiredness and sadness score, as well as in function. Conclusion: Despite late hospitalization and with a large extent of the disease, the participant showed improvement in some symptoms suggesting the benefit of palliative care. In a specialized unit, the symptoms of pain, tiredness and sadness, as well as the functionality of the participants in the weeks of hospitalization. Therefore, these results corroborate the importance of care based on convenience and assistance in the development of public policies and guide the planning of care for the sick.

Descriptors: Palliative Care; Signs and Symptoms; Neoplasms; Nursing.

\section{EGUIMIENTO LONGITUDINAL DEL MANEJO DE SINTOMAS EN SERVICIO ESPECIALIZADO DE ATENCIÓN ONCOLÓGICA PALIATIVA}

Objetivo: Analizar la funcionalidad y los síntomas de los pacientes con cáncer al final de la vida durante la hospitalización en una unidad especializada de atención paliativa. Método: Esta es una evaluación longitudinal desde el ingreso hasta la muerte, con monitoreo semanal de la funcionalidad y la puntuación de los sintomas, de 59 participantes. La prueba de Wilcoxon paramétrica pareada se utilizó para evaluar la mediana de las puntuaciones de los síntomas y la funcionalidad con un nivel de significación del 5\%. Resultados: La muestra del estudio consistió en individuos femeninos, con una edad promedio de 60 años y funcionalidad limitada. Predominó el diagnóstico de cáncer del tracto digestivo. En cuanto a la evaluación de los síntomas y la funcionalidad, considerando la segunda y tercera semana de hospitalización, se observó una mejora en el puntaje de dolor, cansancio y tristeza, así como en la función. Conclusión: A pesar de la hospitalización tardía y con una gran extensión de la enfermedad, el participante mostró una mejoría en algunos síntomas que sugieren el beneficio de los cuidados paliativos. En una unidad especializada, los sintomas de dolor, cansancio y tristeza, asi como la funcionalidad de los participantes en las semanas de hospitalización. Por lo tanto, estos resultados corroboran la importancia de la atención basada en la conveniencia y la asistencia en el desarrollo de politicas públicas y guían la planificación de la atención a los enfermos.

Descriptores: Cuidados Paliativos; Signos Y Síntomas; Neoplasias; Enfermería.

${ }^{1}$ Universidade de Brasília-UNB, DF, Brasil.

Autor correspondente: Karine Marques Costa dos Reis - Email: karinereisdf@hotmail.com

Recebido: 24/03/2020 - Aceiro: 07/08/2020 


\section{INTRODUÇÃO}

O câncer atualmente se destaca entre as afecções crônicas no cenário mundial. No momento, está entre as quatro principais causas de morte no mundo ${ }^{1-2}$. Em 2019, foram 1.762.450 milhões de novos casos de câncer e 606.880 de mortes, sendo essa última com declínio de $1.4 \%$ e $1.8 \%$ ao ano ${ }^{3}$. No Brasil, espera-se 625 mil casos novos, nos próximos dois anos ${ }^{1}$. Diante dessa progressão da doença associado a maior sobrevida, surge nova necessidade de cuidado.

Os cuidados paliativos, como filosofia de cuidado e especialidade técnica, surgiram há seis décadas durante essa transição epidemiológica e demográfica. Nesse sentido, os cuidados paliativos emergem atendendo a uma necessidade de maior cuidado com doenças que ameacem a vida, sendo definida pela IAHPC (International Association for Hospice \& Palliative Care), em 2018, como cuidado multidimensional ativo dos indivíduos com graves sofrimentos relacionados à saúde, independente da faixa etária ${ }^{4}$. Nessa perspectiva, os cuidados paliativos têm como princípio o controle de sintomas, a partir do trabalho interdisciplinar e o cuidado no fim da vida, resgatando o indivíduo como um ser integral e não dissociado de sua família ${ }^{5}$.

Indicado desde o diagnóstico de doença grave, os cuidados paliativos crescem em significado quando não há possibilidade de modificação do curso da doença. Apesar da obrigatoriedade do serviço na rede de atenção ao câncer segundo portaria 483 de 2014, não há, todavia, especificação ou regulamentação para a criação desse serviço ${ }^{7-8}$. Inúmeros estudos randomizados, no entanto, demonstram a efetividade da admissão precoce nessa filosofia de cuidado como resultado de melhor manejo dos sintomas, qualidade de vida e menor custo à saúde a partir de equipe interdisciplinar especializada e com foco no conforto ${ }^{9-10}$.

Coexistindo com a terapia modificadora de doença, os cuidados paliativos têm como objetivo principal o manejo da dor e outros sintomas, principalmente no final de vida. O fato é que apesar da incidência crescente do câncer no país e da necessidade de $80 \%$ desses casos de manejo de sintomas por equipe especialista em cuidados paliativos, o serviço no Brasil ainda é classificado como 3A. Essa classificação traduz um serviço no país como insuficiente e oferecido de modo isolado, ratificado também pelo ranking de qualidade de morte, o qual o Brasil carrega a $42^{\circ}$ posição comparando com 80 países $^{8,11,12}$.
Logo, diante de inúmeros estudos, os quais representam a importância associada a carência de serviços no Brasil, o presente estudo justifica-se ao apontar o período mínimo necessário para admissão e o melhor manejo dos sintomas. Ao profissional enfermeiro, o estudo auxilia e otimiza a assistência, na medida que traz indícios quanto os sintomas mais críticos no manejo. Nesse sentido, o planejamento do enfermeiro e seu cuidado estarão baseados em evidências e prioridades.

Diante de tais dados, realizou-se a seguinte questão norteadora: Como os sintomas e a funcionalidade de doentes oncológicos em fim de vida se comportam durante a internação em unidade de cuidados paliativos? Assim sendo, o objetivo geral desse trabalho foi analisar a funcionalidade e os sintomas de pacientes oncológicos em fim de vida durante internação em unidade especializada em cuidados paliativos.

\section{MÉTODO}

\section{Tipo de Estudo}

Trata-se de avaliação prospectiva, observacional, sem grupo controle, de pacientes oncológicos em fim de vida da admissão até óbito.

\section{Participantes da pesquisa}

A amostra inicial do estudo consistiu em 66 pacientes oncológicos admitidos em unidade de cuidados paliativos exclusivos de fevereiro e agosto de 2018 . Para fim de cálculo amostral, utilizou-se o quantitativo das internações no mesmo período do ano anterior em um teste bicaudal, assumindo o nivel de significância de $5 \%$ e o poder do teste de $80 \%$. A amostra foi selecionada utilizando uma amostragem probabilística aleatória simples, por sorteio, utilizando-se o número da enfermaria e leito a partir do aplicativo Rondomizer.

O critério de inclusão foi estar lúcidos e em condições clínicas de participar plenamente do estudo. Já o critério de exclusão foi: internação em período menor que 48 horas e realização de quimioterapia ou radioterapia paliativa. Inicialmente, foram selecionados 66 participantes, dos quais sete vieram a óbito na primeira semana, impedindo avaliação adicional. Na segunda semana foram seis óbitos, seguidos por 28 na terceira semana e demais óbitos nas oito semanas consecutivas como demonstrado na figura 1. Para fim da análise, consideraram-se a amostra da segunda e terceira avaliação. 
Figura 1. Fluxograma de acompanhamento dos pacientes do estudo - Brasília, 2018.

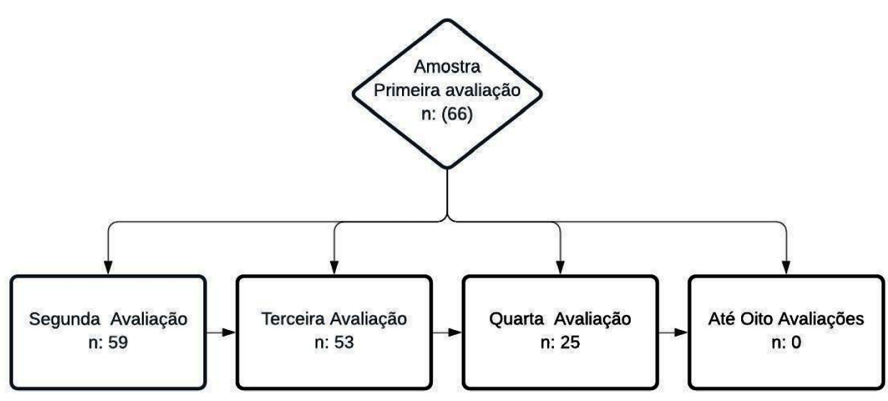

\section{Local de estudo}

Esse estudo foi desenvolvido com pacientes admitidos em unidade hospitalar especializada em cuidados paliativos exclusivos, da secretaria de saúde do Distrito Federal, Brasil. A unidade presta assistência interdisciplinar com foco no conforto, contando com profissionais enfermeiro, fisioterapeuta, médico e nutricionista. Todos especialistas em cuidados paliativos.

O local de estudo, atualmente, consta com 19 leitos, distribuídos em sete enfermarias e taxa de ocupação de $70 \%$. Os pacientes internam a partir de relatório médico e definição de cuidados paliativos exclusivos, já que a equipe não conta com tecnologia pesada para reanimações ou medidas de prolongamento a vida.

\section{Coleta de Dados}

$\mathrm{Na}$ admissão, considerada primeira avaliação, utilizou-se questionário, que abrangia variáveis sociais e demográficas como sexo, idade, estado civil e religião além de aspectos clínicos, avaliados a partir do diagnóstico médico, tempo de diagnóstico e motivo internação. Entre as escalas validadas para o português, que eram aplicados na admissão e semanalmente, estavam a avaliação da funcionalidade através da Escala de Performance Paliativa (PPS) e avaliação dos sintomas utilizando a Escala de Avaliação de Sintomas de Edmonton (ESAS-r).

A escala PPS avalia a funcionalidade e prognóstico, cujo escore varia de 0 a 100. Os parâmetros considerados são mobilidade, atividade e evidências de doenças, autocuidado, ingestão e estado de consciência, onde a pontuação zero evidencia a morte, já 100 representa pequena extensão da doença e total funcionalidade ${ }^{13}$.

Para maior asserção na aplicação do instrumento, foi utilizado o aplicativo desenvolvido pelo Centro de Telessaúde do Hospital das Clínicas da UFMG denominado "Cuidados Paliativos" e disponivel para o download gratuito na Google Play.
Outra escala utilizada foi o ESAS-r, cujos sintomas avaliados são dor, cansaço, sonolência, náusea, apetite, falta de ar, depressão, ansiedade e bem-estar. Na escala, a estratificação da intensidade dos sintomas é numérica, onde zero representa ausência do sintoma e dez a pior sensação possivel ${ }^{14}$.

O procedimento de coleta de dados era realizado semanalmente, às terças feiras no período vespertino, através de visitas beira leito. Para fins de análise dos dados, foram feitas comparações entre a segunda e terceira avaliações.

A funcionalidade e score dos sintomas foram monitorados até o óbito dos pacientes. Todas as aferições foram realizadas pela mesma pesquisadora para maior validade $\mathrm{e}$ uniformização das medidas.

\section{Procedimento de análise e tratamento dos dados}

Inicialmente realizou-se avaliação descritiva da amostra. Na fase analítica do estudo, avaliou-se a distribuição normal de cada variável do ESAS-r e do PPS a partir do teste Kolmogorov Smirnov $(p<0,05)$. Por tratar-se de variável quantitativa contínua não paramétrica utilizou-se o teste Wilcoxon pareado, o qual avalia a mediana das diferenças entre as duas amostras relacionadas. As análises foram realizadas com o programa IBM SPSS (Statistical Package for the Social Sciences) 23.0. O nível de significância utilizado em todo estudo foi de $5 \%$.

\section{Aspectos Éticos}

Preservaram-se os aspectos éticos na presente revisão, onde todos os participantes preencheram o Termo de Consentimento Livre e Esclarecido. A pesquisadora, que também é enfermeira da unidade, realizou seleção, a abordagem dos participantes e coleta de dados. $\mathrm{O}$ estudo é parte integrante do projeto de pesquisa aprovado por meio do parecer número 2.136.574 (Protocolo CAAE no 66315117.0.0000.0030).

\section{RESULTADOS}

Em relação às características sociodemográficas e clínicas apresentadas pelos 66 participantes iniciais deste estudo, o sexo predominante foi o feminino (52\%), com idade média de 58,64 anos, baixa escolaridade $(62,1 \%)$. Entre a indicação de internação predominante, a dor predominou (46,9\%), seguida de dispneia (24,2\%). 0 diagnóstico médico prevalente foi o tumor do aparelho digestório com $36,3 \%$. Quanto ao tempo do diagnóstico e tempo de internação, a mediana foi de 15 meses e 16 dias respectivamente. 
Tabela 1. Caracterização sócio demográfica e clínica de pacientes internados em instituição pública do Distrito Federal. Brasilia, DF, Brasil, 2018. $(n=66)$

\begin{tabular}{|c|c|c|}
\hline Variáveis & $n$ & $\%$ \\
\hline \multicolumn{3}{|l|}{ Sexo } \\
\hline Feminino & 34 & $52 \%$ \\
\hline Masculino & 32 & $48 \%$ \\
\hline \multicolumn{3}{|l|}{ Escolaridade } \\
\hline Fundamental & 41 & $62 \%$ \\
\hline Analfabeto & 13 & $20 \%$ \\
\hline Médio & 11 & $17 \%$ \\
\hline Graduação & 1 & $2 \%$ \\
\hline \multicolumn{3}{|l|}{ Estado Civil } \\
\hline Casado & 31 & $47 \%$ \\
\hline Solteiro & 15 & $23 \%$ \\
\hline Divorciado & 12 & $18 \%$ \\
\hline Viúvo & 8 & $12 \%$ \\
\hline \multicolumn{3}{|c|}{ Indicação de internação } \\
\hline Dor & 29 & $44 \%$ \\
\hline Náusea/vômito & 7 & $11 \%$ \\
\hline Dispneia & 12 & $18 \%$ \\
\hline Sangramento & 5 & $8 \%$ \\
\hline \multicolumn{3}{|c|}{ Diagnóstico Médico - Sítio Primário } \\
\hline Gastrintestinal & 24 & $36 \%$ \\
\hline Reprodutivo & 19 & $29 \%$ \\
\hline Mama & 15 & $23 \%$ \\
\hline Respiratório & 3 & $5 \%$ \\
\hline Mama & 3 & $5 \%$ \\
\hline Cabeça e pescoço & 2 & $3 \%$ \\
\hline
\end{tabular}

Quanto a avaliação prospectiva dos sintomas e funcionalidade, observou-se que as variáveis dor, cansaço, tristeza, bem como o ESAS-r total e o PPS foram significativos ao nível de significância de $5 \%$. Em todos os casos, observa-se uma diminuição significativa do score sintoma na terceira avaliação em relação à segunda, já a avaliação de funcionalidade manteve a pontuação, com aumento no intervalo de confiança (Tabela 2).
Tabela 2. Análise de associação dos sintomas constantes no ESAS-r (em separado), ESAS-r total e PPS em relação às avaliações 2 e 3 realizadas no acompanhamento de pacientes internados em unidade especializada em Cuidados Paliativos, 2018.

\begin{tabular}{|c|c|c|c|c|c|c|c|}
\hline & \multicolumn{3}{|c|}{ Avaliação 2} & \multicolumn{3}{|c|}{ Avaliação 3} & \multirow[b]{2}{*}{$p^{*}$} \\
\hline & $\mathrm{n}$ & $\begin{array}{l}\text { Me- } \\
\text { diana }\end{array}$ & Ia & $\mathrm{n}$ & $\begin{array}{l}\text { Me- } \\
\text { dia- } \\
\text { na }\end{array}$ & Ia & \\
\hline ESAS Total & 59 & 45,0 & 23,5 & 53 & 30,0 & 36,0 & $<0,001$ \\
\hline Dor & 59 & 6,0 & 4,0 & 53 & 4,5 & 4.0 & 0,006 \\
\hline Cansaço & 59 & 6,0 & 5,0 & 53 & 4,0 & 4.3 & 0,040 \\
\hline Sonolência & 59 & 6,0 & 4,0 & 53 & 4,0 & 6,0 & 0,157 \\
\hline Náusea & 59 & 2,0 & 5,0 & 53 & 2,0 & 4,3 & 0,661 \\
\hline Apetite & 59 & 8,0 & 5,0 & 53 & 6,0 & 4,0 & 0,428 \\
\hline Dispneia & 59 & 2,0 & 5,0 & 53 & 2,0 & 4.0 & 0,114 \\
\hline Tristeza & 59 & 6,0 & 6,0 & 53 & 6,0 & 6,0 & 0,016 \\
\hline Ansiedade & 59 & 6,0 & 4,5 & 53 & 5,0 & 6.0 & 0,202 \\
\hline Bem-estar & 59 & 4,0 & 2,5 & 53 & 4,0 & 4,5 & 0,165 \\
\hline PPS & 59 & 40,0 & 20,0 & 53 & 40,0 & 30,0 & 0,004 \\
\hline
\end{tabular}

* Teste de Wilcoxon de amostras relacionadas.

A variável sintomas, representadas pelos componentes do ESAS-r, apresentaram diferença estatisticamente significativa. Essas são apresentadas através do Box plot nos dois momentos, onde a linha negrita representa a mediana, devendo-se destacar que o estudo não apresentou valores discrepantes ou outlier (Figura 2).

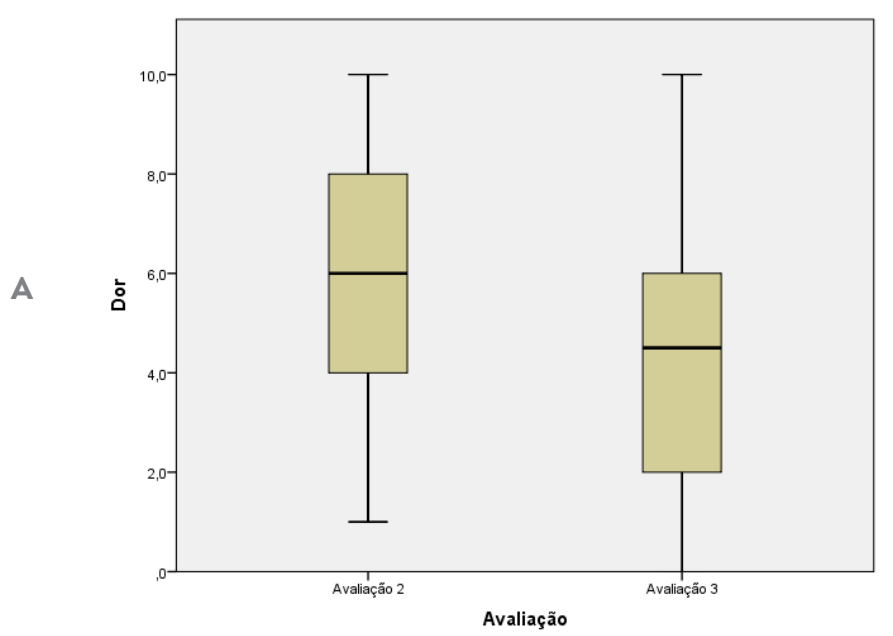


B
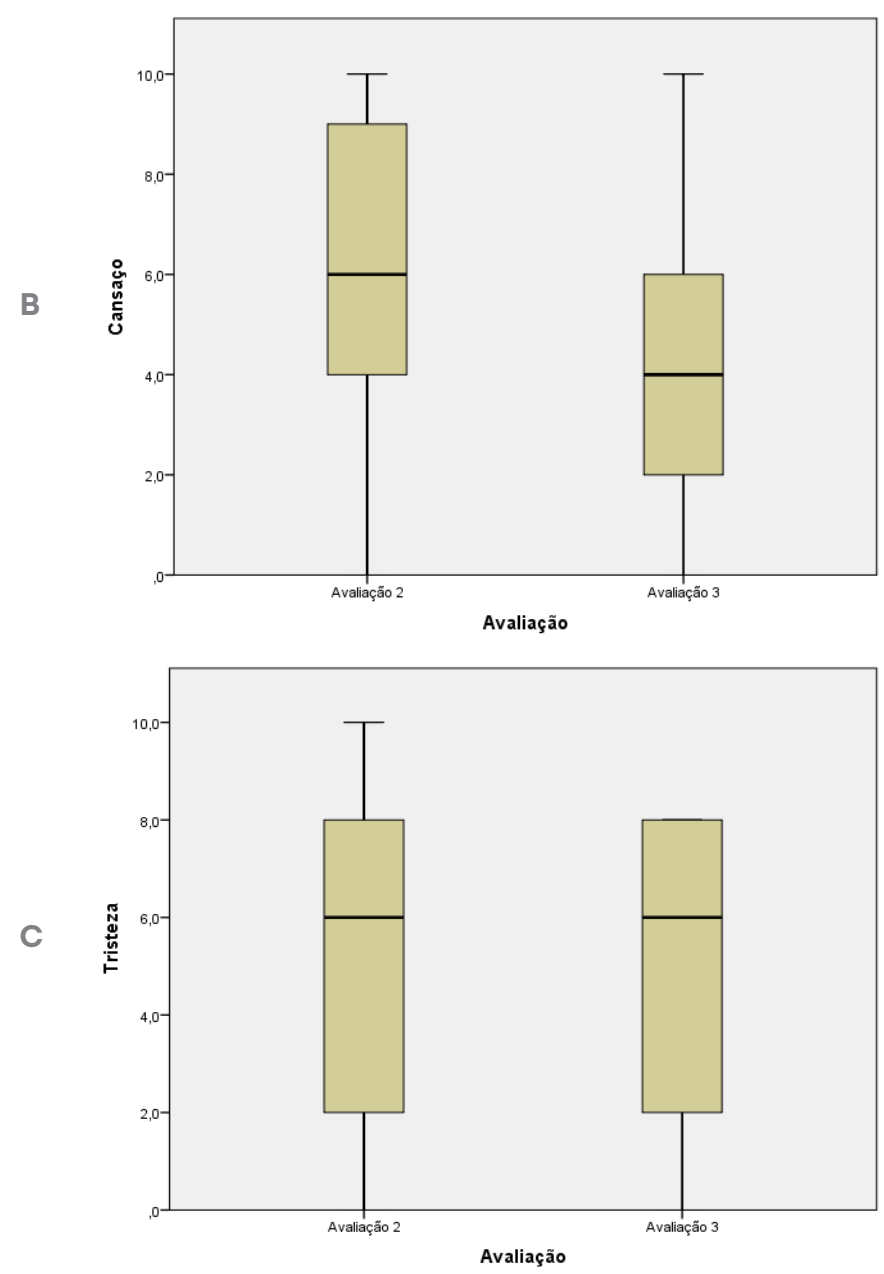

Figura 2. Box plot das variáveis ESAS sintomas significativamente diferentes nas avaliações 2 e 3 (a - dor, b - cansaço, c-tristeza) realizadas em pacientes internados em unidade especializada, 2018.

\section{DISCUSSÃO}

Os achados sociais, demográficos e clínicos corroboram com estudo de avaliação multidimensional a partir do diagnóstico de enfermagem. Nesse estudo, houve predominância feminina, com idade entre 60 a 69 anos, casados, católicos, ensino fundamental incompleto e diagnóstico médico de câncer gastrointestinal ${ }^{15}$.

Quanto aos sintomas com necessidade de manejo, evidenciou-se nas 59 avaliações prospectivas viáveis, que em uma semana houve melhora do ESAS total, como também do ESAS dor, cansaço, tristeza e piora na avaliação da funcionalidade. Todos com um desfecho inversamente proporcional, onde o aumento dos dias de cuidado (internação) resultou melhora da funcionalidade e score dos sintomas.

O provável benefício dessa tecnologia leve de cuidado na melhora qualidade de vida a partir do manejo dos sintomas, demonstrado pelo ESAS-r total, é ratificado em estu- do brasileiro. Esse revelou que o acompanhamento de indivíduos no fim de vida, a partir dos cuidados paliativos teve associação positiva entre a variável clínica tempo de hospitalização, qualidade de vida e score da escala de sintomas ${ }^{16}$.

Ao avaliar os sintomas separadamente, observa-se que o componente ESAS-r para a dor apresentou mediana de 6,0 decrescendo para 4,5 na segunda semana de internação. É sabido que a dor é o sintoma mais comum no fim de vida, o qual gera maior sofrimento e desconforto, estando presente em $85 \%$ dos pacientes em cuidados paliativos exclusivos $^{17-18}$.

Esse sintoma, no entanto, apesar da prevalência, tem alta possibilidade de controle farmacológico ${ }^{17-18}$. Há também, a partir desse julgamento clínico, medidas não farmacológicas e intervenientes para o manejo efetivo e avaliação positiva da resposta às intervenções ${ }^{19}$. Nesse sentido, o estudo sugere a eficiência da filosofia dos cuidados paliativos, que trás como prioridade o manejo e controle dos elementos da dor, no sentindo de reduzir o sofrimento e garantir maior bem-estar ${ }^{20}$.

Ainda sobre o manejo da dor, um estudo retrospectivo de 40 indivíduos portadores de doença oncológica observou redução do score da dor em 48 horas, porém sem significância. Houve, entretanto, melhora efetiva nas últimas 48 horas de vida, onde os acompanhantes percebem tranquilidade e conforto ${ }^{21}$. Logo, todo esse referencial, corrobora com a presente pesquisa e denota a importância do cuidado por equipe interdisciplinar em individuos no fim da vida.

Outro sintoma com melhora significativa após duas semanas de internação foi o componente cansaço da escala ESAS. Tão prevalente e incômodo como a dor, a fadiga em estudo transversal com 386 individuos ocorreu em 32,8\% da amostra, sendo o sintoma com maior repercussão na qualidade de vida ${ }^{22}$.

Diante da multidimensionalidade e subjetividade do sintoma, a fadiga além de frequente é um dos sintomas mais debilitantes, traduzindo na menor funcionalidade e sobrevida, sendo descrita como uma sensação de cansaço agu$\mathrm{da}^{23}$. Nesse sentido, diante da importância clínica esperava-se melhor manejo desse sintoma, o que de fato ocorreu inclusive repercutindo na funcionalidade. Há, entretanto, dúvidas que requerem maiores pesquisas sobre as estratégias efetivas proporcionadas para a melhora do cansaço, já que esse sintoma tem limitação do tratamento e desconhecimento dos mecanismos fisiopatológicos ${ }^{23}$.

Em outro estudo, que avalia sintomas diferentes da dor a tristeza se destaca. Como sintoma do domínio psicoespiritual, a tristeza é resultado das perdas funcionais, o es- 
tresse existencial e a busca de significado diante de uma doença grave ${ }^{24}$. O que chama atenção é a manutenção do score da tristeza. A significância no manejo do sintoma em duas semanas, porém, ocorreu pela diminuição do intervalo de confiança, mesmo com a ausência do profissional psicólogo.

Deve-se atentar que no presente trabalho, os profissionais que têm maior contato com paciente, a partir da detecção do sofrimento, manejo farmacológico a partir da administração de medicações, cuidados com o corpo durante a vida e após a morte é o profissional enfermeiro. Assim, corroborando com a tese da importância do enfermeiro no manejo dos sintomas, em estudo internacional, cujo objetivo foi demonstrar a importância das relações de cuidado do profissional enfermeiro, indica que a atividade da equipe de enfermagem é essencial para o manejo de necessidades psicoespirituais ${ }^{25}$.

Conforme as outras variáveis, a funcionalidade também apresentou melhora no escore. Deve-se atentar, no entanto, que no presente estudo os pacientes chegavam tardiamente na unidade de internação. Os participantes tinham funcionalidade média de 30 , o que representa dependência completa, ingesta reduzida, totalmente acamado e consciência completa ou sonolência. Deve-se destacar, todavia que de acordo com estudo, o melhor intervalo de tempo para admissão nos cuidados paliativos exclusivos é de seis meses que antecedem a morte ${ }^{26}$. Já na presente pesquisa, os pacientes acessavam o serviço nas últimas semanas de vida, contrariando recomendações. O manejo dos sintomas, no entanto, era alcançado parcialmente junto com a funcionalidade e autonomia, traduzindo na possivel efetividade desse cuidado mesmo diante da lentidão na admissão.

\section{Limitações do Estudo}

Diante da metodologia prospectiva, observacional e analítica, o presente estudo tem bom nível de evidência. A ausência do grupo controle, no entanto, nos impede de associar a melhora clínica com a admissão no serviço, sendo a principal limitação do estudo.

\section{Contribuições do estudo para a prática}

O presente estudo indicou melhora dos sintomas no fim de vida de pacientes oncológicos, o que sugere maior efetividade da assistência em cuidados paliativos. Assim, contribui à medida que estimula a elaboração de políticas públicas específicas como também a criação fluxo de atendimentos ou até serviços especializados para essa população. Além disso, o estudo auxiliará na tomada de decisão do profissional enfermeiro quanto os sintomas com maior propensão de melhora e os de maior complexidade. Haverá também maior consciência sobre a necessidade do acolhimento, cuidado integral, como também o manejo da pessoa em detrimento da doença.

Todo esse cuidado ao indivíduo, portanto, transformará o morrer, valorizando o bem-estar e a qualidade de vida. Outrossim, a relevância do estudo emerge ao inferir a importância da participação do profissional enfermeiro diante do cuidado, o que pode ser de interesse para pesquisas futuras sobre intervenções do profissional enfermeiro na dor, cansaço e tristeza.

\section{CONCLUSÃO}

Diante do objetivo do estudo, indivíduos no fim de vida com doença oncológica apresentaram melhora dos sintomas dor, tristeza, cansaço e funcionalidade, apesar da progressão da doença. Tal alívio, foi possivel diante de duas semanas de internação, o que sugere a alta efetividade do serviço na proposta de confortar durante a terminalidade. $\mathrm{Na}$ presença desses achados, o estudo poderá contribuir na concepção de políticas públicas que regulamentem o serviço como também no planejamento do cuidado do enfermeiro.

\section{Contribuição dos autores:}

Karine Marques Costa dos Reis: concepção e desenho do estudo de revisão, busca em base de dados, análise dos resultados, discussão e conclusão. Cristine Alves Costa de Jesus: concepção e desenho do estudo e última análise crítica do artigo antes da submissão.

\section{REFERÊNCIAS}

1. Instituto Nacional de Câncer José Alencar Gomes da Silva [Internet]. Estimativa 2020: incidência de câncer no Brasil. 2020 [cited 2020 Jun 20]. 120 p. Available from: https://www.inca.gov.br/sites/ufu.sti.inca. local/files/media/document/estimativa-2020-incidencia-de-cancer-no-brasil.pdf.

2. Bray F, Ferlay J, Soerjomataram I, Siegel RL, Torre LA, Jemal A. Global cancer statistics 2018: GLOBOCAN estimates of incidence and mortality worldwide for 36 cancers in 185 countries. CA Cancer J Clin [Internet] 2018 [cited 2020 Jun 20] ;68(6):394-424. Available from: http://www.fasgo. org.ar/images/Bray-et-al-2018-CA-A-Cancer-Journal-for-Clinicians.pdf. 
3. Siegel R, Ward E, Jemal A. Cancer Statistics, 2019. CA Cancer J Clin [Internet]. 2019 [cited 2020 Jun 20]:57(1):43-66. Available: from: https:// acsjournals.onlinelibrary.wiley.com/doi/epdf/10.3322/caac.21551.

4. International Association for Hospice \& Palliative Care. Palliative Care Definition. [Internet] 2018. [cited 2020 Jun 20]. Available from: https://hospicecare.com/what-we-do/projects/consensus-based-definition-of-palliative-care/definition/.

5. Hui D, Bruera E. Integrating palliative care into the trajectory of cancer care. Nature Reviews Clinical Oncology. 2015; 13:159-171.

6. Downar J, Wegier P, Tanuseputro P. Early Identification of People Who Would Benefit From a Palliative Approach-Moving From Surprise to Routine. JAMA. 2019;2(9):e1911146.

7. Ministério da Saúde (BR). Portaria $n^{\circ} 483$, de $1^{\circ}$ de abril de 2014 . Redefine a Rede de Atenção à Saúde das Pessoas com Doenças Crônicas no âmbito do Sistema Único de Saúde (SUS) e estabelece diretrizes para a organização das suas linhas de cuidado. [Internet] 2014. [cited 2020 Jun 20]. Diário Oficial União, 2 abril 2014, Seção 1,p.71. 120 p. Available from: https://bvsms.saude.gov.br/bvs/saudelegis/gm/2014/ prt0483_01_04_2014.html.

8. Academia Nacional de Cuidados Paliativos. Análise Situacional e Recomendações Para Estruturação De Programas De Cuidados Paliativos No Brasil [Internet]. Academia Nacional de Cuidados Paliativos. 2018 [cited 2020 Jun 20]. 28 p. Available from: https://paliativo.org. $\mathrm{br} / \mathrm{wp}$-content/uploads/2018/12/ANALISE SITUACIONAL_ANCP18122018.pdf

9. Greer JA, Tramontano AC, McMahon PM, Pirl WF, Jackson VA, ElJawahri A, et al. Cost Analysis of a Randomized Trial of Early Palliative Care in Patients with Metastatic Nonsmall-Cell Lung Cancer. J Palliat Med [Internet]. 2016 [cited 2020 Jun 20];19(8):jpm.2015.0476. Available from: http://online.liebertpub.com/doi/10.1089/jpm.2015.0476.

10. Becker CL, Arnold RM, Park SY, Rosenzweig M, Smith TJ, White DB et al. A cluster randomized trial of a primary palliative care intervention (CONNECT) for patients with advanced cancer: Protocol and key design considerations. Contemp Clin Trials. 2017:54:98-104.

11. The Economist Intelligence Unit. The 2015 Quality of Death Index Ranking palliative care across the world. The Economist [Internet] 2015 [cited 2020 Jun 20];71. Available from: http://www.apcp.com.pt/ uploads/2015-EIU-Quality-of-Death-Index-Oct-6-FINAL.pdf

12. Santos CE, Peixoto Caldas JM, Serafim JA, Barros N. Needs for Palliative Care of Cancer Patients in Brazil: Analysis of Data from 2008-2014. J Palliat Care Med [Internet]. 2017 [cited 2020 Jun 20];07(01):1-8. Available from: https://www.omicsgroup.org/journals/ needs-for-palliative-care-of-cancer-patients-in-brazil-analysis-ofdata-from-20082014-2165-7386-1000294. php?aid=84020.

13. Sanvezzo VM de S, Montandon DS, Esteves LSF. Instruments for the functional assessment of elderly persons in palliative care: an integrative review. Rev Bras Geriatr e Gerontol [Internet]. 2018 [cited 2020 Jun 20]:21(5):604-15. Available from: https://www.scielo.br/pdf/rbgg/ v2ln5/1809-9823-rbgg-21-05-00604.pdf
14. Monteiro DR, Almeida MA, Kruse MHL. Tradução e adaptação transcultural do instrumento Edmonton Symptom Assessment System para uso em cuidados paliativos. Rev Gaúcha Enferm [Internet]. 2013 [cited 2020 Jun 20];34(2):163-71. Available from: https://www.scielo.br/ pdf/rgenf/v34n2/v34n2a21.pdf.

15. Xavier É de CL, Junior AJSC, Carvalho MMC, Lima FR, Santana ME. Nursing diagnoses in oncology palliative care according to the multidimensional approach diagram. Enferm foco [Internet]. 2019 [cited 2020 Jun 20];10(3):152-7. Available from: http://revista.cofen.gov.br/index. php/enfermagem/article/view/2109/569.

16. Freire MEM, Costa SFG da, Lima RAG de, Sawada NO. Health-related quality of life of patients with cancer in palliative care. Texto Context - Enferm [Internet]. 2018 [cited 2020 Jun 20]:27(2):1-13. Available from: https://www.scielo.br/pdf/tce/v27n2/0104-0707-tce-27-02-e5420016. pdf.

17. Kelley AS, Morrison RS. Palliative care for the seriously ill. N Engl J Med. 2015;373(8):747-55

18. Worldwide Palliative Care Alliance. Global Atlas of Palliative Care at the End of Life [Internet]. 2014 [cited 2020 Jun 20]. 103 p. Available from: https://www.who.int/nmh/Global_Atlas_of_Palliative_Care.pdf.

19. Sousa ADRS, Silva LF, Cavalcanti ACD, Góes FGB, Moraes JRMM. Palliative care nursing care instrument for pediatric oncological intensive care center. Enferm foco [Internet]. 2019 [cited 2020 Jun 20]:10(7):28-34. Available from: http://revista.cofen.gov.br/index.php/ enfermagem/article/view/2436/546

20. Bhatnagar S, Gupta M. Integrated pain and palliative medicine model. Ann Palliat Med [Internet]. 2016 [cited 2020 Jun 20]:5(3):196-208. Available from: http://apm.amegroups.com/article/ view/10504/11572.

21. Campbell CL, Kelly M, Rovnyak V. Pain management in home hospice patients: A retrospective descriptive study. Nurs Health Sci. 2017:19(3):381-7.

22. Li B, Mah K, Swami N, Pope A, Hannon B, Lo C, et al. Symptom Assessment in Patients with Advanced Cancer: Are the Most Severe Symptoms the Most Bothersome? J Palliat Med. 2019:XX(XX):1-8.

23. Dean A. The holistic management of fatigue within palliative care. Int J Palliat Nurs. 2019;25(8):368-76.

24. Hagmann C, Cramer A, Kestenbaum A, Durazo C, Downey A, Russell M, et al. Evidence-based Palliative Care Approaches to Nonpain Physical Symptom Management in Cancer Patients. Semin Oncol Nurs [Internet]. 2018 [cited 2020 Jun 20]:34(3):227-40. Available from: https://doi.org/10.1016/j.soncn.2018.06.004.

25. Vbnml C, Iranmanesh S, Nayeri ND, Tirgari B, Kalantarri B. Compassion and care at the end of life: oncology nurses' experiences in South-East Iran. a Int J Palliat Nurs. 2016:22(12):588-97.

26. Finn L, Malhotra S. The Development of Pathways in Palliative Medicine: Definition, Models, Cost and Quality Impact. Healthcare. 2019;7(1):22 